\title{
Incurred sample reproducibility: 10 years of experiences: views and recommendations from the European Bioanalysis Forum
}

\author{
Morten Anders Kall ${ }^{1}$, Marco Michi², Barry van der Strate ${ }^{3}$, Achim Freisleben ${ }^{4}$, Daniela \\ Stoellner ${ }^{5}$ \& Philip Timmerman*,6 \\ ${ }^{1} \mathrm{H}$ Lundbeck A/S, Copenhagen, Denmark \\ ${ }^{2}$ Aptuit an Evotec company, Verona, Italy \\ ${ }^{3}$ PRA Health Sciences, Assen, The Netherlands \\ ${ }^{4}$ Nuvisan GmbH, Grafing, Germany \\ ${ }^{5}$ Novartis Institutes for BioMedical Research, Basel, Switzerland \\ ${ }^{6}$ European Bioanalysis Forum, Brussels, Belgium \\ *Author for correspondence: chair@e-b-f.eu
}

With 10 years of experiences on incurred sample reanalysis (ISR) as an integrated part of regulated bioanalysis, the European Bioanalysis Forum has reflected on the implementation and the use of ISR. Three surveys were conducted in 2016 and 2017 as a revisit of the ISR experiences within European pharmaceutical industry and contract research organizations: has ISR become a tool for postvalidation and process check of a bioanalytical method performance and has ISR become a routine in our laboratories? Do we agree on the interpretation of guidelines/guidance and are we aligned in our approach - among others?

First draft submitted: 19 July 2018; Accepted for publication: 27 September 2018; Published online: 6 November 2018

Keywords: European Bioanalysis Forum • incurred sample reanalysis • incurredsample reproducibility

Incurred sample reanalysis (ISR) was, in its current form, introduced at the AAPS Spring Workshop in Crystal City, 1-3 May 2006 (CC III) and the recommendations were later published in 2007 [1]. This CC III White Paper was rapidly followed by several publications; Fast et al. [2], the EBF ISR recommendations [3] and recommendations from the third Calibration and Validation Group Workshop [4]. Later, these publications were followed up by the European Medicines Agency (EMA) guideline (EMEA/CHMP/EWP/192217/2009) to be effective in 2011 (rev \#1 corr. 2 from 2015) [5]. The draft US FDA guidance to industry was published in 2013 [6], followed in 2014 by the Crystal City V report [7], recommendations from the Global Bioanalysis Consortium [8] and Japanese Ministry of Health, Labour and Welfare (MHLW) Guideline on Bioanalytical Method Validation in Pharmaceutical Development [9].

Following their initial ISR recommendation paper from 2009 [3], the European Bioanalysis Forum (EBF) has conducted three major surveys in 2016 and 2017 as a revisit of the ISR experiences within European pharmaceutical industry and contract research organization (CROs). The results from the surveys were discussed during internal EBF meetings and subsequently presented at international conferences (AAPS workshop, NJ, USA, 13-15 September 2017, EBF Focus Workshop, Lisbon, Portugal, 24-26 September 2017 and the 10th EBF Open Symposium 15-17 November 2017, Barcelona, Spain). Throughout the discussions and the surveys, a significant amount of data were generated and some, but not all, data are shared in this publication. The focus of the surveys was on the general understanding and approach toward handling of ISR in regulated bioanalysis. Based on the data and feedback, some refined recommendations on ISR are provided in this publication. There is a general agreement among EBF members that conduct of ISR creates value as a postmethod validation parameter when applied to those studies currently called out for in guidelines/guidance. However, there was also a general understanding among EBF members, that ISR is performed more often and in more studies than called for in the guidelines/guidance. There was a general agreement that ISR criteria are clear on pass/fail, however the scope for study selection is not clear 
for all. Additionally, the guidelines/guidance on handling single samples with large variations in a passed ISR run are not clear.

One major driver throughout the discussions was how to interpret different aspects of the guidelines/guidance - do we, as industry, do what is asked for or do we conduct more ISR than is expected from the regulators?

Attention was brought to the frequency of the reported ISR failures, which was shown to be very low $(\sim 1.4 \%)$. Aspects of ISR failures will be discussed, for example, when were ISR failures most frequent, in which kinds of studies and what was the cause for failure, and what was the impact?

The current FDA guidance made effective from May 2018 [10] was not part of the discussions and evaluation. However, the expectations from the FDA 2018 guidance are discussed in line with other guidelines and White Papers.

\section{Method}

All three surveys with focus on experiences with ISR were submitted to the EBF community over a 12-month period and results were presented and discussed at internal EBF meetings and conferences. The surveys were designed to obtain quantitative measurements, but also to receive open feedback with individual comments. The first survey received feedback from 30 member-companies, representing Pharma and CRO in the ratio 17:13, LC-MS and ligand binding assays (LBA) in the ratio 24:16. Ten member-companies replied on behalf of both their LC-MS and LBA groups. The results from the first survey were presented and discussed at an internal EBF meeting that was held in connection to the EBF Barcelona conference in November 2016.

The second survey received feedback from 33 member-companies, equally representing Pharma and CRO (17:15), LC-MS and LBA (24:18), and again here nine members provided feedback from both their LC-MS and LBA groups. The data were presented and discussed at an internal EBF members meeting in the spring 2017. Based on the discussions it was decided to continue the ISR evaluation and to conduct yet another survey.

A third survey was conducted during summer 2017 and was focused on the preparation of feedback to the ICH M10 joined AAPS/EBF/JBF sister meetings in fall of 2017. The first meeting was the AAPS workshop in NJ, USA, 13-15 September 2017: "Towards global harmonization of bioanalytical method validation" followed by the EBF focus workshop: "Industry input into ICH M10: experimental data as the cornerstone for a science-driven bioanalytical guideline" Lisbon, Portugal, 24-26 September 2017. And finally, at the 10th EBF Open Symposium: "A New Journey Begins" 15-17 November 2017, Barcelona, Spain.

The third survey received feedback from 30 member-companies where three replied both for their LC-MS and LBA groups, with a little skewed distribution between LC-MS and LBA (21/12). The number of feedback may reflect some 'survey fatigue' that appeared within the EBF community during summer 2017 after comprehensive feedback requests in preparation of the ICH M10 workshops.

\section{Results}

The first survey was focused on the scope of ISR, while the second and the third surveys were focused on causes of failed ISR; for example, in which study types did ISR failed, and what procedures did the laboratories have place to handle failed ISR - among others. The surveys also aimed to identify aspects or phrasings in the current guidelines/guidance that would not be sufficiently unambiguous to serve as clear guidance. A few aspects with some degree of ambiguity were identified. For instance, it is mentioned in the EMA guideline, section 6, that "Large differences between results may indicate analytical issues and should be investigated." However, it was not clear what constitutes a large difference in a single sample, especially when ISR passes. Additionally, the survey revealed that it was not clear for all how, for example, to define a clinical study is 'pivotal' with regard to bioanalysis. Regarding this point and in consideration to the recommendation from the FDA, it was not clear how to select samples around $\mathrm{C}_{\max }$ and elimination phase for all those 'pivotal Phase III studies' where the entire time profile is not sampled and only few time points may be collected for population PK. Another unclear aspect was if ISR needs to be assessed in new trials in patients in case a new therapeutic indication is explored. On the other hand, it was the feedback from a majority that the EMA Guideline in general is very clear.

Hence, numerous aspects of the ISR conduct were discussed several times as the understanding of these issues matured. The following review of the discussions and generated data may not reflect in the chronological order in which they were discussed but they are rather stacked in categories of related aspects. 


\section{Scope of ISR}

There is a general agreement within the EBF community that ISR is a strong analytical tool for postvalidation assessment of reproducibility and robustness of the bioanalytical method when applied to study samples, and that the conduct of ISR creates value.

From the first survey, it was the understanding that ISR was being conducted more often than expected by the regulatory authorities and that ISR may be used as a 'process check' or as an additional justification for a dataset as kind of in-study validation. Considering that ISR could simply confirm wrong results in a reproducible way, the question was raised if ISR is indeed the proper tool for this process check. Nevertheless, it was also acknowledged that there are no well-established alternative approaches to ISR.

Few argued that ISR did not add value in their setup, however when looking at the data related to these statements (e.g., biomarkers, in vitro assays), these types of analysis would not be in scope for ISR in the current guideline. With the current FDA 2018 guidance [10], this may however change in the future.

The understanding of the scope of ISR was more ambiguous. Approximately $60 \%$ would conduct ISR in PK studies only, however, 50\% indicated that they would also conduct ISR in studies with population PK and non-PK studies. These figures made it difficult to understand the general perception as such, however the written feedback did reveal that the ISR approach may have been very different within the EBF group. Some Pharma companies did conduct ISR for all (clinical) studies in the development program, others kept it strictly to the guidelines/guidance.

Both CRO and sponsor should agree on the approach to be taken on ISR.

\section{The ISR approach}

As part of the ISR revisit, it was important to understand the variations and unity in the approaches to ISR within the EBF community: how the guidelines/guidance have been interpreted and implemented.

According to the EMA guideline [5] and the EBF recommendations [3], ISR should be conducted at least in the following situations:

- Toxicokinetic studies once per species;

- All pivotal bioequivalence trials;

- First clinical trial in subjects;

- First patient trial;

- First trial in patients with impaired hepatic and/or renal function.

\section{Selection of studies}

Almost $50 \%$ of EBF members stated that they, for different reasons, would conduct ISR in more studies/situations than mentioned above. Examples mentioned were to conduct ISR in all clinical studies when PK is the primary end point, drug interaction studies, studies with unusual patient populations or disease states, or studies with special populations (i.e., pediatrics/geriatrics and special geographical regions, etc.). Additional study types mentioned were pivotal biomarker assays, preclinical PK studies of final formulation in animal health projects and PK studies of new routes of administration.

Approximately $30 \%$ of EBF members indicated that they would conduct ISR in all regulated bioanalysis studies (nonclinical and clinical), for monitoring of the entire bioanalytical process.

These additional studies where ISR were conducted may reflect that most labs have based their ISR approach, not only on the EMA guideline, but in addition implemented aspects of other ISR guidance. The papers mentioned in addition to the EMA guideline were the EBF recommendations [3] (22\%), parts of the CCIII paper [1] (52\%), parts of the 2013 draft FDA Guidance [6] (11\%), parts of GBC recommendations [8] 11\% and finally $4 \%$ had implemented parts of the CCV recommendation paper [4].

One aspect that is not mentioned in the EMA guideline was the conduct of ISR following major method changes and/or after revalidation of the method.

\section{ISR after revalidation}

The EBF ISR paper [3] recommends to perform ISR 'upon major method changes' and 'first-time use of an excising method in a new laboratory'. This was also addressed in the recommendations on ISR by Viswanathan [1] formulated as assessment of ISR 'once per method/species/laboratory'. 
Despite the clear recommendations from EBF and Viswanathan to reset the ISR approach upon method changes, only approximately $40 \%$ would restart ISR after change and modification of a method. A total of $50 \%$ reported a case-by-case approach and 10\% did not repeat ISR with a revalidated method. A total of $10 \%$ had a written procedure to define how to handle ISR after revalidation: which degree of method change would imply a new method and consequently justify the restart of the ISR approach. Most had a case-by-case approach based on scientific judgment, however a consolidated feedback from the obtained data would be that if the method changes were significant, for example, changes of selectivity or sample processing method (solid phase extraction [SPE] vs protein precipitation), then the ISR approach should be restarted.

\section{Documentation of ISR}

Is ISR a post- or in-study validation parameter and should ISR data be amended to the bioanalytical method validation report or reported together with the bioanalytical data from the study where ISR has been conducted? ISR is related to the performance of a particular bioanalytical method but ISR data apply to a specific study or a phase, so how is a track kept of the postvalidation ISR data?

From the perspective of a reviewer, it may be helpful to understand why ISR was, or was not, conducted in a particular study. Was ISR conducted to comply with guidance (first time in species, population, matrix or a new method, or first in new laboratory), was it conducted to add confidence to data? or was ISR was conducted with the same method in a previous study? Approximately $30 \%$ would, at the current time of surveys, state the purpose of ISR in each study protocol or (phase) report and almost $50 \%$ would make a reference to a previous study where ISR was conducted.

It is recommended, although not required in the guideline/guidance, to keep good documentation practices and justify the decision of performing ISR in a study - or not.

\section{Aberrant results in passed ISR tests}

One issue that promoted discussions from the start of the first survey was how to handle the expectations from the EMA guideline on large differences in results in passed ISR runs phrased as: "large differences between results may indicate analytical issues and should be investigated".

Approximately $30 \%$ had at the time of survey, written procedure on how to define and handle the above and how to trigger an investigation in case of aberrant/different results in accepted ISR experiments. Many had a case-by-case approach but no general agreement on how to define 'large differences' was revealed from the survey. The following tiered approach can however be consolidated from the feedback: single aberrant results ( $>30 \%$ deviation) should not trigger any repeat analysis in case of accepted ISR experiments - unless they form a trend. Hence, in such a case it is recommended to justify why no further action was taken.

It is also recommended to define the terms within each laboratory for situations with a passed ISR run, where there is a presence of systematic bias for several results (e.g., $>30 \%$ ) or a limited number of results with a strong bias (e.g., $>50-60 \%$ ). What investigation should be conducted to identify the possible causes of this bias and, if necessary, how to implement corrective actions. Again, if an investigation is conducted, it is recommended to use good documentation practice.

\section{Investigation on ISR failure rates}

It is stated in the EMA guideline: "in case incurred sample analysis shows deviating results, this should be investigated, and adequate steps should be taken to minimize inaccuracy (and precision)." Two-third of the members had implemented written procedures for root cause analysis in case of failed ISR experiments, but only approximately a third had written procedures for how to handle failing root cause analysis for failed ISR (that is: if no cause of failure can be identified).

The below tiered approach can however be consolidated from the feedback:

If human or technical error can be excluded for reason of ISR being outside acceptance criteria, then it is necessary to:

- Identify cause (e.g., discrepancies may be due to metabolite interaction, metabolite/product interconversion and matrix effects);

- Define and propose possible corrective actions, including revalidation and/or reanalysis of all study samples; 


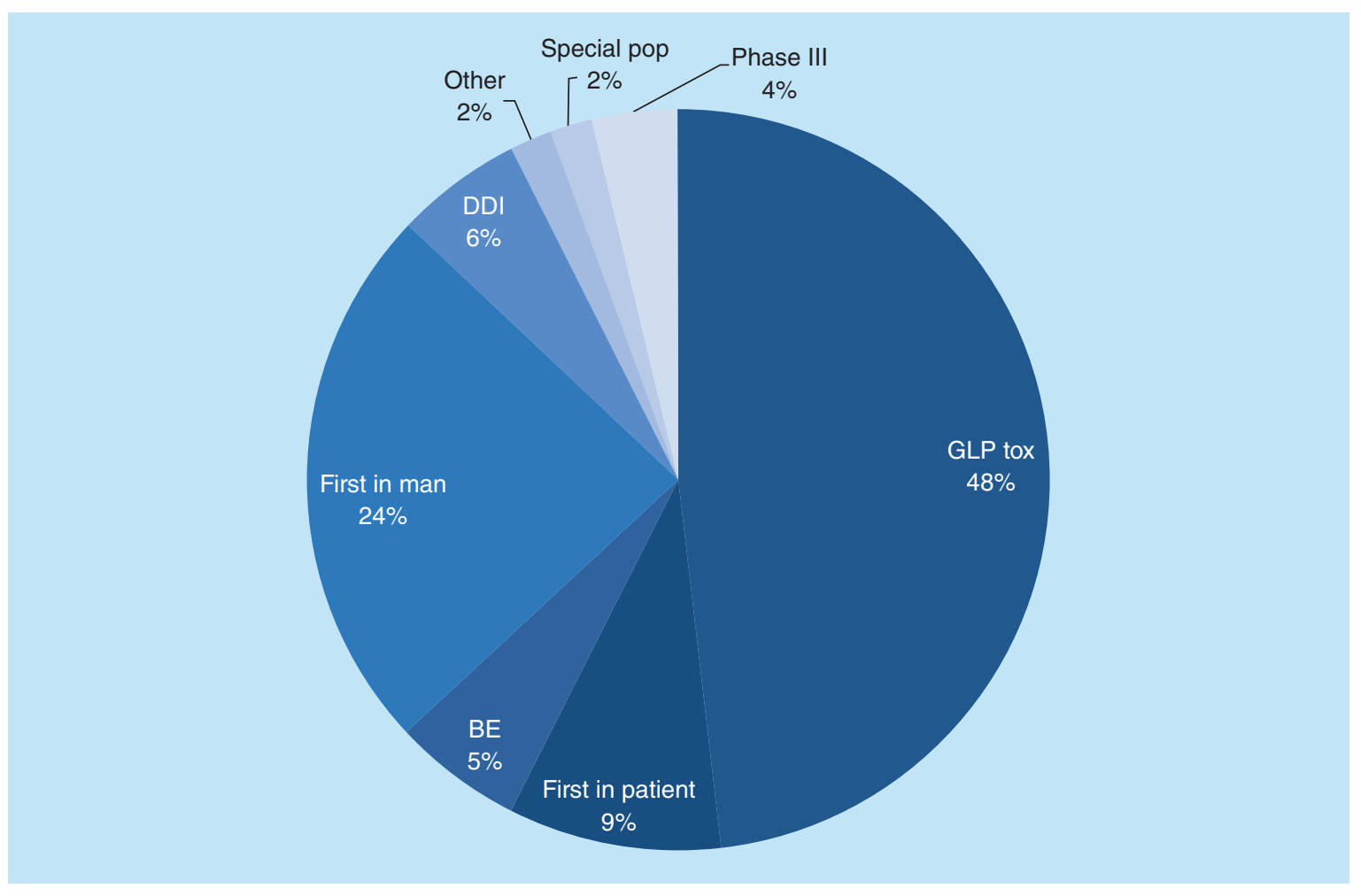

Figure 1. The distribution of study types where failed incurred sample reanalysis was reported. In a total of 3951 studies with regulated bioanalysis, conducted by 32 laboratories, 54 studies were reported with a failed incurred sample reanalysis. The main impacted studies were GLP toxicity studies (48\%), first-in-man (24\%), first-in-patient $(9 \%), \mathrm{BE}(5.5 \%), \mathrm{DDI}(5.5 \%)$, Phase III (4\%), special population ( $2 \%)$ and others $(2 \%)$.

BE: Bioequivalence study; DDI: Drug-drug interaction study; GLP: OECD Good Laboratory Practice.

- Conduct an impact assessment, discuss with relevant parties (e.g., sponsor, study director or clinical trial leader), restart analysis when applicable.

If no cause of failure can be identified, every step of the investigation and their outcome must be documented. It is recommended to apply good documentation practice and document the conclusions of the investigation, including an assessment of the reliability of the data and corrective action for future studies - among others.

\section{Frequency of failed ISR, cause \& impact}

A principal argument for conducting ISR was formulated in 2007 by Viswanathan et al. [1]:

"Spiked standards and quality control samples may in many situations not sufficiently mimic study samples from dosed subjects due to presence of metabolites that can be converted to the parent species, differences in protein binding in patient samples, recovery issues, sample inhomogeneity and mass spectrometric ionization matrix effects, etc."

Hence, IRS is related to the method selectivity rather than reproducibility issues related to human or technical errors, analyte instability - among others. This argument was later adopted by the EMA guideline [5].

In the EBF surveys, data were collected on the frequency of failed ISR, the cause of failures, the impact of failures and in which type of studies ISR failures most frequently had been observed (Figure 1).

In survey 2, ISR data from 5563 studies conducted as part of regulated bioanalysis were reported. The reported average ISR failure rate was $1.4 \%$ and the difference in ISR failures between LC-MS analysis and LBA was imperceptible (1.1 vs $1.7 \%)$.

The survey was not designed to provide data that were sufficiently detailed to draw strong conclusions on reasons for ISR failures, however, analytical errors and instability issues were the major causes of the reported ISR failures. 


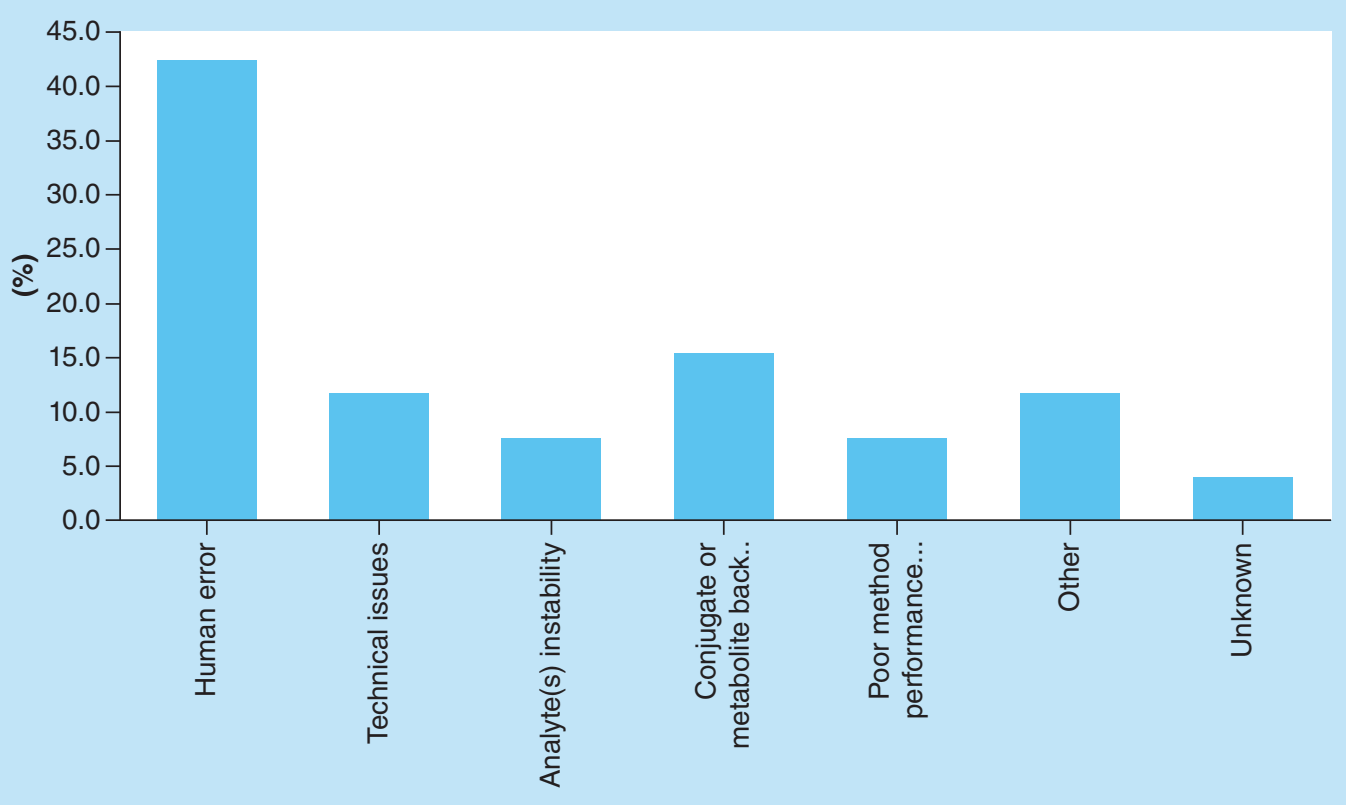

Figure 2. The distribution of the cause of failed incurred sample reanalysis in the Good Laboratory Practice toxicity studies where failed incurred sample reanalysis was reported. The major cause of failed incurred sample reanalysis in the OECD Good Laboratory Practice toxicity studies (Figure 1) was human errors, which corresponded to $42 \%$. Technical errors $(12 \%)$, analyte- $(8 \%)$ or conjugate instability $(15 \%)$ and poor method performance, including selectivity issues $(8 \%)$, and others $(15 \%)$.

Poor method performance that could be related to selectivity issues only attributed to approximately $5 \%$ of the $1.4 \%$ failed studies (e.g., 4/5563 studies).

To investigate this, and to get a better resolution of data, an additional survey was conducted. The new dataset generated in survey 3 was based on 3951 regulated studies conducted by 32 laboratories and here 54 studies out of 3951 studies were reported with a failed ISR corresponding to a failure rate of $1.4 \%$ which was the same as in the first survey.

The main impacted studies (48\%) were OECD Good Laboratory Practice (GLP) toxicity studies, followed by first-in-man (24\%), first-in-patient (9\%), bioequivalence study (BE; 5.5\%), drug-drug interaction study (DDI; $5.5 \%)$, Phase III (4\%) special population (2\%) and others (2\%).

The major cause of failed ISR in the GLP toxicity studies was human errors, which corresponded to $42 \%$ of the total number of GLP studies considered. Remaining causes for ISR failure were: technical errors (12\%), analyte $(8 \%)$ or conjugate instability (15\%) and poor method performance, including selectivity issues (8\%) and others (15\%) (Figure 2).

For the data for first-in-man (13 failed ISR), first-in-patient ( 5 failed ISR), BE (3 failed ISR), DDI (3 failed ISR) and special population ( 1 failed ISR), the size of the dataset would not justify dividing these numbers into subgroups and generate data on distributions of failure cause. However, one clear trend was that the frequency of human and technical error would decrease as the studies were progressing into late clinical development, and that the frequency of poor method performance issues increased along the same order. Hence, the decrease in human/technical error could be a result of experience and the assay becoming more routine as the program progresses. Poor method performance could be that the assay is less suitable for analysis as complexity increases with program progression: diseased matrices, comedication - among others (Figure 3).

To evaluate the impact of the failed ISR studies and within each category, the failed studies were divided into three groups based on the applied approach:

- After failed ISR, stop analysis, investigate cause of failures and resume analysis when solved;

- After failed ISR, stop analysis, investigate cause of failures, optimize method and conduct revalidation of method, resume analysis; 


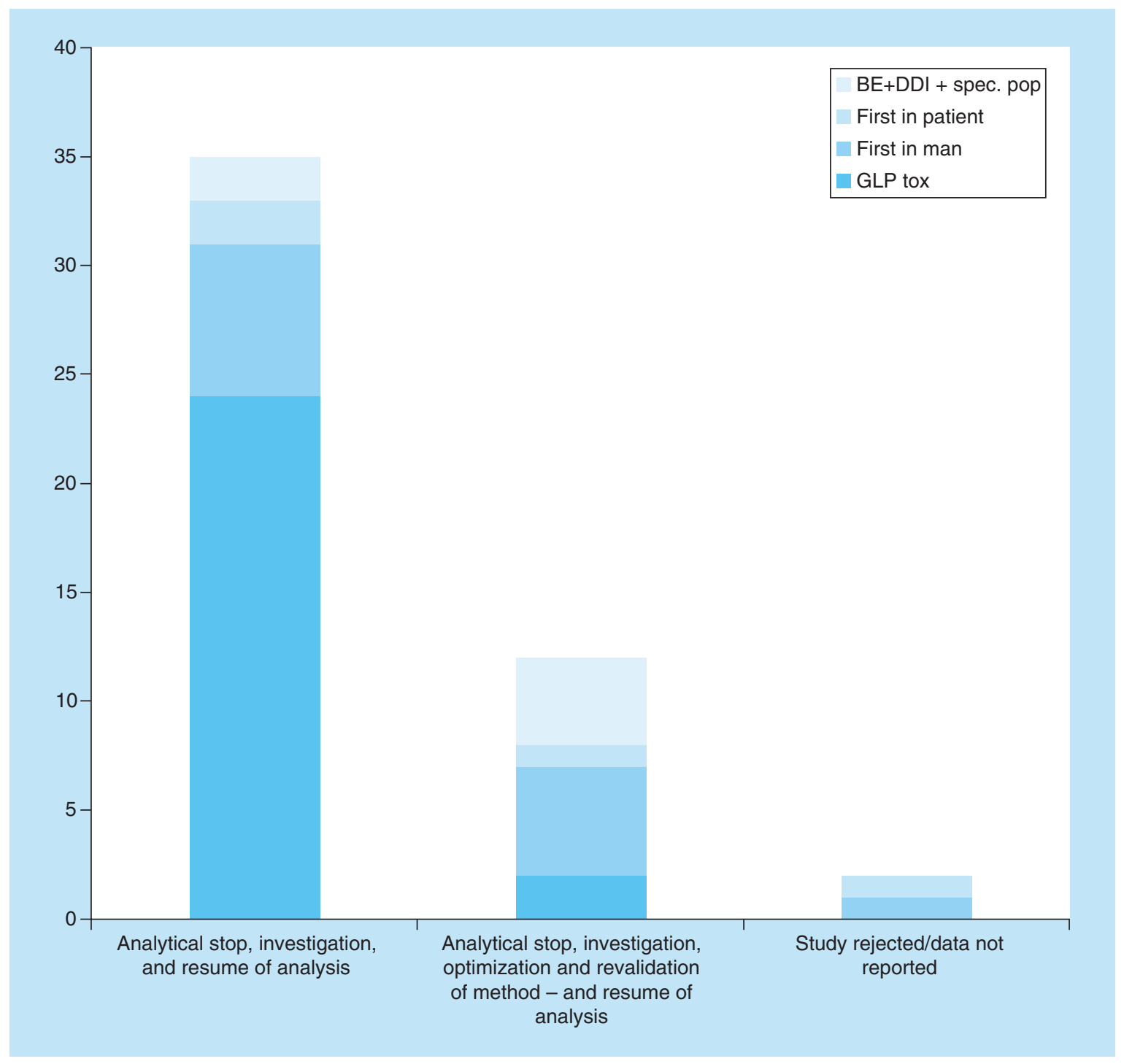

Figure 3. The impact of the failed incurred sample reanalysis studies were divided into three groups based on how the failures were managed. In $70 \%$ of the studies with failed incurred sample reanalysis (ISR), the cause of failures was resolved and the analysis was resumed without any modification of the method or procedures. In $25 \%$ of ISR failures, the method or procedures had to be changed and revalidated before the analysis could be resumed. In $5 \%$ of the failed studies, the data were rejected due to failed ISR. The color code indicates the distribution of studies into four main groups of studies: GLP toxicity studies, first-in-man, first-in-patient and pivotal pharmacokinetics studies. BE: Bioequivalence study; DDI: Drug-drug interaction study; GLP: OECD Good Laboratory Practice.

- After failed ISR, stop analysis study, investigate cause of failures, reject data or data not reported.

In 70\% of the studies with failed ISR, the cause of failures was resolved and the analysis was resumed without any modification of the method or procedures. In $25 \%$ of ISR failures, the method or procedures had to be changed and revalidated before the analysis could be resumed and finally in $5 \%$ of the failed studies, the data were rejected due to failed ISR.

\section{Sponsor-CRO relations}

A recurrent point of view from CRO members was that on one side, ISR should be conducted to comply with the requirements from the sponsor and at the same time to comply with the expectations from authorities.

There was a general agreement among the EBF members that the ISR approach applied by the CRO should always be decided based on mutual agreement between sponsor and CRO. 


\section{Discussion}

Looking at the guidance on ISR in general, the scope of ISR can be summarized to an evaluation of the reproducibility of bioanalytical data generated in pharmacokinetic, bioequivalence and preclinical safety studies. The survey data indicate, however, that industry performs ISR more often than called for in the guidelines/guidance, even though the failure rates continue to be low. Although still infrequent, the majority of failed ISR occurs in early GLP toxicity studies, but data indicate that the nature and the magnitude of the cause of ISR failures, would not be impacting safety decisions. The question remains if the current frequency of ISR conducted by industry adds additional value or if the quality of data would be the same if we limit the frequency as per the guidelines/guidance?

ISR was implemented as an evaluation of the method reproducibility when analyzing incurred samples because such samples would challenge the method more than spiked quality controls (QCs) and standards. The data from 10 years' experience have clearly shown that ISR, even though the failure rate is very low, acts as an in-study validation parameter or as part of the QC of an analytical method or an analytical run. The conduct of ISR adds confidence to data. This is corroborated by the fact that the majority of failed ISR was reported to be due to human or technical errors. These failures were, for unknown reasons, not identified by the in-study validation process control of QCs, dilution QCs, carryover tests or blanks. This was not part of the surveys, but one could speculate that the implementation of ISR successfully has increased the awareness of the need of highly selective methods for pivotal PK studies over the last 10 years and hence eliminated or at least decreased the reason for implementing ISR. On the other hand, a very low ISR failure rate was reported already in 2009 by the EBF [3]. From the present data, it can be argued that, implementation of ISR seems to have had the desired effect of increasing the focus on method robustness and selectivity in pivotal PK studies within (European) industry, because very few ISR failures were reported for such studies in our surveys and the reported failed ISR in pivotal studies (BE, DDI, PK special population) did not impact the study.

With the current dataset available, it can be justified to reduce the aimed sample size for ISR in future guidelines/guidance to a total of $5 \%$, irrespective of the size of a study.

The scope of ISR in the EMA guideline on study selection is for the majority, unambiguously clear. Situations where additional ISR should be conducted from a scientific point of view have been outlined in recommendation papers $[1,8]$ and the present manuscript provides more detailed and operational recommendation on how to handle these. However, despite the above results, it was the experience from the surveys that many laboratories execute ISR as an in-study validation parameter/process control in studies and numbers not called for in the guideline, at increased cost for the overall study sample analysis.

ISR and ISR-like experiments, are excellent tools for in-study validation/process control to add confidence in data. For instance, in large BE studies some would argue to apply ISR for each subject in the study, rather than strictly following the guidelines/guidance, to eliminate the business risk of failures. Nonregulated bioanalysis conducted within the boundaries of a scientific validation [12] may benefit from the conduct of ISR-like experiments to add confidence into the method performance and hence the data. However, in order to avoid promoting expectations from the regulators that ISR should be conducted in a large veracity of studies beyond what is mentioned in the guidelines/guidance, the bioanalytical scientists should consider clearly to state the purpose for the additional ISR or that these ISR-like experiments are conducted within a scientific validation approach.

ISR is a postvalidation of a specific method that is applied to postdose study samples from a specific study or in other words, a validation parameter that is evaluated after bioanalysis of postdose samples. Throughout a development program, it may be necessary to adjust a method. Hence, it is important to define when the method has been adjusted to a degree where the method should be regarded as a new method - and where ISR data from the previous studies no longer can be applied as postvalidation of the updated, new method. Written procedure as to when to reset the ISR approach after major method changes and revalidation should be considered as well as a system to keep track of in which studies, which methods have been applied on ISR.

The single-sample variation in passed ISR mentioned in the EMA guideline was discussed at several occasions and it was obvious that this was not easy to deal with. A deviation of up to $30 \%$ for LC-MS analysis or $40 \%$ for LBA between two single analyses in a repeat analysis of study samples would in most cases be accepted as a confirmation of first result [8]. So, what constitutes a large difference in a single sample in a passed ISR run and what should trigger an investigation? We recommend to use a tiered approach where a single or few aberrant results ( $>30 \%$ deviation) should not trigger any repeat analysis - unless they form a trend. The presence of systematic 
bias (>30\%) or a strong bias ( $>50-60 \%)$ should trigger an investigation. In all cases, it is recommended to justify the choices made.

Throughout the discussions during the internal EBF meetings and at conference meetings, it became clear that the scope for study selection for ISR was not clear. The term 'pivotal' as used by the FDA has caused some uncertainty on how to understand this in relation to bioanalysis, and this uncertainty may have lead ISR been conducted more than called for in the guideline/guidance. A clear and narrow scope for ISR would help the industry to conduct ISR in the right studies with an optimal benefit of ISR.

In the MHLW bioanalytical method validation guideline from 2013 [9], the scope for ISR is formulated in a clear way: "Usually, ISR is performed for representative studies selected for each matrix in studies that use pharmacokinetic data as the primary end point."

In the recent FDA 2018 bioanalytical guidance to industry [10], the scope for ISR is formulated as: "ISR should be conducted in all studies submitted in an new drug application (NDA), biologic license applications (BLA) or abbreviated new drug applications (ANDA) that provide pivotal data for the approval or labeling of the product, regardless of the matrix. For instance, ISR is expected for all in vivo BE studies in ANDA, or all pivotal pharmacokinetic, pharmacodynamic and biomarker studies in NDAs or BLAs."

It may be difficult to understand how one single study fits into the above requirements in a large development plan with duration of more than 10 years, and to understand what conclusions will be drawn based on the bioanalytical data - eventually in a submission. This may be even more difficult to understand for a CRO without an open dialog and shared information between sponsor and the CRO.

With the FDA 2018 guidance, biomarkers have been introduced to the scope of ISR and a new revisit of industry experience with this broader scope may become relevant in the near future.

\section{Conclusion: recommendations on ISR}

- Limit the number of studies and study types with ISR as a regulatory requirement;

- Use ISR experiments as an in-study validation function parameter if needed to add confidence to data, but explicitly state why this was conducted;

- Use ISR-like experiments for nonregulated bioanalysis as in-study validation/process control/incurred sample stability - among others - but avoid using the term ISR;

- Reset the ISR approach after revalidation of method changed/updated that affect the method selectivity or sample processing method (e.g., SPE to protein precipitation);

- Use a tiered approach to handle aberrant results in passed ISR tests where single aberrant results (e.g., >30\%) do not trigger any further investigation -unless they formed a trend. As an example, a trend could be identified by the presence of systematic bias $(>30 \%)$ or a strong bias ( $>50-60 \%)$. Irrespective of the decision, it is recommended to handle this according to written procedures and to justify the applied approach.

\section{Future perspective}

ISR has, now 10 years after its introduction, become an integrated part of regulated bioanalysis. The implementation and interpretations of guidance has been discussed at several occasions and in many publications. The scope of ISR has been a challenge for many bioanalytical laboratories, and many would argue that the scope is too broad to serve as a clear guidance. In spring 2018, the FDA issued an updated guidance to industry on Bioanalytical method validation, and a global ICH M10 Bioanalytical Method Validation guideline is expected to be issued in 2019. With these two new guidance in place, the scope of ISR may become more focused or the scope of ISR may become broader, to cover a larger variety of studies. In both cases, industry will work together to implement the new guidance and eventually a new revisit of industry experience with this new scope of ISR may become relevant.

\section{Acknowledgements}

The authors acknowledge the European Bioanalysis Forum member companies that have contributed to the surveys, workshops and internal discussions, which form the backbone of the current recommendation.

\section{Disclaimer}

The views and conclusions presented in this paper are those of the European Bioanalysis Forum and do not necessarily reflect the representative affiliation or company's position on the subject. 


\section{Financial \& competing interests disclosure}

The authors have no relevant affiliations or financial involvement with any organization or entity with a financial interest in or financial conflict with the subject matter or materials discussed in the manuscript. This includes employment, consultancies, honoraria, stock ownership or options, expert testimony, grants or patents received or pending, or royalties.

No writing assistance was utilized in the production of this manuscript.

\section{References}

1 Viswanathan CT, Bansal S, Booth B et al. Workshop/conference report - quantitative bioanalytical methods validation and implementation: best practices for chromatographic and ligand-binding assays. AAPS J. 9(1), E30-E42 (2007).

2 Fast DM, Kelly M, Viswanathan C et al. AAPS workshop on current topics in GLP bioanalysis: assay reproducibility for incurred samples - implications of crystal city recommendations. AAPS J. 11(2), 238-241 (2009).

3 Timmerman P, Luedtke S, van Amsterdam P et al. Incurred sample reproducibility views and recommendations by European Bioanalytical Forum. Bioanalysis 1(6), 1049-1056 (2009).

4 Savoie N, Garofolo F, van Amsterdam P et al. 2009 white paper on recent issues in regulated bioanalysis from the 3rd calibration and validation group workshop. Bioanalysis 2(1), 53-68, (2010).

5 EMA, European Medicines Agency. Guideline on bioanalytical method validation. EMEA/CHMP/EWP/ 192217/2009 (2011). https://www.ema.europa.eu/documents/scientific-guideline/guideline-bioanalytical-method-validation_en.pdf

6 US FDA, US Department of Health and Human Services. Draft guidance for industry: bioanalytical method validation (2013). http://academy.gmp-compliance.org/guidemgr/files/UCM368107.PDF

7 Booth B, Arnold ME, DeSilva B et al. Workshop report: Crystal City V - quantitative bioanalytical method validation and implementation: the 2013 revised FDA guidance. AAPS J. 17(2), 277-288 (2015)

8 Fluhler E, Vazvaei F, Singhal P et al. Repeat analysis and incurred reanalysis: recommendations for best practices and Harmonisation from the Global Bioanalysis Consortium Harmonisation team. AAPS J. 16 (6),1167-1174 (2014).

9 Japanese Ministry of Health, Labour and Welfare. Guideline on bioanalytical method validation in pharmaceutical development. MHLW, Japan (2013). http://www.nihs.go.jp/drug/BMV/250913_BMV-GL_E.pdf

10 US FDA. Guidance for Industry: Bioanalytical Method Validation. US Department of Health and Human Services, US FDA, Center for Drug Evaluation and Research. Rockville, MD, USA (2018). https://www.fda.gov/downloads/drugs/guidances/ucm070107.Pdf

11 Viswanathan CT. Incurred sample reanalysis: a global transformation. Bioanalysis 3(23), 2601-2602 (2011)

12 Timmerman P, White S, McDougall S et al. Tiered approach into practice: scientific validation for chromatography-based assays in early development - a recommendation from the European Bioanalysis Forum. Bioanalysis 7(18), 2387-2398 (2015). 